\title{
O Teste de Zulliger-SC: avaliação da criatividade em crianças
}

\author{
The Zulliger-SC Test: Assessment \\ of children's creativity
}

\author{
Raquel Rossi TAVELLA ${ }^{1}$ \\ Anna Elisa de VILLEMOR-AMARAL²
}

\begin{abstract}
Resumo
Este trabalho teve como objetivo verificar se os indicadores cognitivos e afetivos no Teste de Zulliger - que, por hipótese, se correlacionam com a criatividade -, contribuem para diferenciar crianças com diversos potenciais criativos, identificadas pelo Teste de Criatividade Figural Infantil. Participaram do estudo 90 crianças, de 11 e 12 anos, de ambos os sexos, matriculadas em escolas públicas de uma cidade do interior de São Paulo. A aplicação dos instrumentos foi dividida em duas etapas, na primeira das quais foi aplicado aquele Teste de forma coletiva. A segunda etapa foi realizada individualmente, tendo como instrumento o Teste de Zulliger adaptado para o Sistema Compreensivo, desenvolvido por Exner para o Rorschach. Os resultados apontaram diferenças significativas nas variáveis movimento, movimento ativo, qualidade formal incomum, resposta popular e número de respostas. Conclui-se que as variáveis do Zulliger selecionadas foram sensíveis para identificar crianças com diferentes níveis de criatividade.
\end{abstract}

Palavras-chave: Criança; Criatividade; Teste de Zulliger.

\begin{abstract}
The aim of this study was to verify whether the cognitive and affective indicators in the Zulliger Z Test that theoretically correlate with creativity, help to differentiate children with different creative potential, identified using the Child Figural Creativity Test. The study sample included 90 children of both sexes, aged 11 and 12, from public schools in a city in the countryside of São Paulo State. The application of the instruments was divided into two phases; in the first one the Child Figural Creativity Test was applied collectively. The second step was carried out on individuals and the instrument used was the Zulliger Z Test adapted for the Comprehensive System. The results showed significant differences in the following variables: movement, active movement, unusual formal quality, popular response and response number. It was concluded that the selected variables of the Zulliger Z Test were sufficiently sensitive to enable the identification of children with different levels of creativity.
\end{abstract}

Keywords: Child; Creativity; Zulliger Z Test.

$\boldsymbol{\nabla} \boldsymbol{\nabla} \boldsymbol{\nabla}$

1 Centro Universitário Nossa Senhora do Patrocínio, Faculdade de Saúde e Ciências da Vida, Curso de Psicologia. Salto, SP, Brasil.

2 Universidade São Francisco, Programa de Pós-Graduação em Psicologia. R. Alexandre Rodrigues Barbosa, 45, Centro, 13251-900, Itatiba, SP, Brasil. Correspondência para/Correspondence to: A.E. VILLEMOR-AMARAL. E-mail: <anna.villemor@saofrancisco.edu.br>. 
A avaliação psicológica infantil é um processo bastante complexo, pois esse período da vida é marcado por fases do desenvolvimento que possuem características muito distintas. Os recursos utilizados para avaliar os aspectos cognitivos e afetivos em cada fase devem ser apurados, para que possam auxiliar na identificação de potencialidades e dificuldades da criança (Nascimento, Pedroso \& Souza, 2009). O desenvolvimento de pesquisas na área da avaliação psicológica infantil é fundamental, pois, com base nos resultados das avaliações, poderão ser feitos encaminhamentos e orientações mais adequados, aumentando a possibilidade de que a criança se desenvolva de forma equilibrada (Pérez-Ramos, 2000).

Entretanto, são considerados escassos os instrumentos de avaliação da personalidade infantil que possuam as exigências de rigor científico. Essa carência torna necessário o desenvolvimento de pesquisas que tenham por finalidade averiguar as propriedades psicométricas dos instrumentos psicológicos infantis, fundamentados em metodologias científicas adequadas (Villemor-Amaral, 2006; 2008; Villemor-Amaral \& Pasqualini-Casado, 2006).

Sendo assim, o foco desta pesquisa recairá sobre o estudo de validade de critério do Teste de Zulliger no Sistema Compreensivo (SC). Como critério, foi utilizado o construto criatividade, já que se supõe que esta pode se refletir nas respostas e estar associada com a produtividade da criança no teste. A seleção desse critério deve-se ao fato de a produção de respostas no Zulliger, do mesmo modo que no Rorschach, método que the deu origem, estar associada à criação de ideias, bem como à relação entre as mesmas, na medida em que, para solucionar essa tarefa, o indivíduo deve escolher partes da mancha e decidir com o que elas se assemelham.

Pode-se considerar que esse processo também inclui a formação de impressões perceptivas do estímulo e a comparação com registros mnêmicos (Weiner, 2000). Sendo assim, a produção de respostas no Teste de Zulliger envolve atenção, percepção, tomada de decisão, análise, ou seja, ele490 mentos componentes do funcionamento cognitivo, naturalmente envolvidos na manifestação da criatividade (Sakamoto, Lapastini, \& Silva, 2003; Weiner, 2000).

Devido a sua complexidade, a criatividade tem sido analisada por abordagens filosóficas, biológicas, psicológicas, evolutivas e psicoeducacionais (Wechsler, 1998). No entanto, pode-se verificar que existe um consenso entre estudiosos de que a criatividade deve ser considerada a partir de um modelo multidimensional (Wechsler, 2001) que extrapola o dado cognitivo. Desse modo, a criatividade pode ser compreendida como o resultado da "interação entre processos cognitivos, ambiente, características da personalidade e elementos inconscientes" (Wechsler, 1998, p.91). Nesse mesmo sentido, Oliveira e Alencar (2012) e Barreto e Martinez (2007) argumentam sobre a importância do ambiente escolar para o desenvolvimento da criatividade. Em outra perspectiva, Alencar e Fleith (2003) afirmam que os fatores intrapsíquicos - estilo de pensamento, personalidade e motivação -, são extremamente importantes para que o indivíduo possa usufruir de seu potencial criativo. No entanto, também ressaltam que os fatores socioculturais, tais como valores e regras sociais, contribuem para a manifestação da criatividade, ou até mesmo para sua contenção. Para (Sakamoto, 2000), que se apoia na teoria de Winnicott, as relações afetivas e o ambiente proporcionam a segurança, condição considerada fundamental para a manifestação criativa, já que afetividade e liberdade de expressão são aspectos essenciais para o desenvolvimento e exercício da criatividade. Nessa mesma perspectiva, Barreto e Martinez (2007) afirmam que a manifestação da criatividade é o resultado da associação de componentes afetivos e motivacionais na realização de uma atividade.

Em uma ampla revisão de literatura nas bases de dados Scientific Eletronic Library Online (SciELO) e Biblioteca Virtual em Sáude - Psicologia (BVS-Psi), não foram encontrados estudos recentes que investigassem o teste de Zulliger com crianças, nem tampouco a criatividade. Já no método de Rorschach são inúmeras as investigações que focam a criatividade. Autores como Adrados (1978), Exner e Sendín (1999), Sakamoto et al. (2003) e Weiner (2000) já destacaram as respostas de movimento 
humano como representantes da criatividade. Isso se deve primeira-mente ao fato de que atribuir movimento é uma pura projeção da pessoa que responde ao teste, já que as figuras são estáticas. Mas não se trata apenas do fenômeno da projeção, já que o movimento também pode ser projetado em animais (FM) ou objetos e forças da natureza (M), sem que esses determinantes sejam indicados na literatura como representantes de criatividade. É possível inferir que o movimento humano esteja atrelado a processos criativos, mais do que outros tipos de movimento, justamente em razão dos elementos de maior controle sobre a fantasia e do pensamento deliberado, o que não se encontra na produção de movimento animal ou inanimado (Exner \& Sendín, 1999; Weiner, 2000).

Entretanto, algumas pesquisas levaram a resultados aparentemente contrários. Torres (2010) realizou uma pesquisa com a finalidade de encontrar relação significativa entre imaginação criativa, frequência e qualidade das respostas de movimento em uma amostra de pintores. Os resultados indicaram que tanto a produção como a diversidade das respostas de movimento foram consideradas abaixo da expectativa esperada, não havendo relação entre a produção do determinante movimento e imaginação criativa dos sujeitos. Entretanto, o autor não explicitou quais foram os critérios para a seleção de artistas, tampouco o que considerou imaginação criativa, não havendo também um grupo de comparação que possibilitasse maior segurança quanto aos resultados. Mas, com base em seus achados, o autor concluiu que a imaginação criadora não pode ser avaliada apenas pelas respostas de movimento humano no Rorschach, devendo ser considerados outros aspectos da personalidade avaliados pelo teste. Nesse aspecto, o próprio Rorschach (1978) já enfatizava que uma resposta criativa não pode pertencer a um único determinante, mas envolve uma combinação de fatores, ou seja: grande número de respostas de movimento, diversas respostas de cor, boa qualidade formal, porcentagem reduzida de conteúdo animal e atividade associativa sugerem imaginação criativa.

Dudek (1968) constatou que os indivíduos que apresentam um elevado número de respostas de movimento humano $(M \geq 5)$ demonstram ter mais facilidade em expressar a criatividade do que aqueles que dão um baixo número de respostas de movimento humano $(\mathrm{M} \leq 2)$. As respostas dos indivíduos foram analisadas e formaram dois grupos, um com alta frequência de respostas de movimento e outro com baixa frequência de $M$. Também foi aplicado o Teste de Apercepção Temática (TAT), Figure Drawings e o Mosaic Patterns. Os resultados revelaram que o grupo com maior número de $\mathrm{M}$ no Rorschach demonstrou mais facilidade em expressar criatividade que o grupo com baixo $M$, nos três instrumentos utilizados. Para finalizar, pode-se também verificar que a quantidade de $M$ se correlaciona significativamente com a quantidade de resposta global (W) e com soma ponderada de cor (Sum C), no grupo que possui M elevado. Nessa mesma direção, Joseph e Pillai (1986) realizaram um estudo, por meio da análise dos protocolos do Método de Rorschach e de um teste de pensamento criativo, com alunos de pós-graduação. Verificaram que a resposta de movimento humano foi considerada como uma medida significativa para diferenciar indivíduos com alto e baixo nível de criatividade.

Raychaudhuri (1971) já havia procurado relacionar a produção de respostas de movimento humano no Rorschach com as variáveis sexo, criatividade e orientação psicossexual. Encontrou que o aumento das respostas de movimento humano estava associado com a criatividade e a feminilidade em indivíduos do sexo masculino. Mas, um ano depois, em outro trabalho, reconsiderou seus resultados em vista de problemas na composição de sua amostra e, em novo artigo, afirmou não ser possível concluir, com base na pesquisa anterior, que haveria diferença entre sexo, orientação sexual e produção de respostas de movimento humano no Rorschach (Aronow \& Raychaudhuri, 1972).

Singh, Majhi e Singht (2007) realizaram um estudo com a finalidade de verificar a validade do Teste de Rorschach como medida de criatividade. Participaram do estudo 100 indivíduos não pacientes, de ambos os sexos, na faixa etária de 18 a 20 anos. Também foi utilizado nesse estudo o Teste Verbal do Pensamento Criativo. Os resultados indicam que os grupos dos indivíduos mais e menos 
criativos diferem significativamente em número de respostas (R), W e resposta popular (P). Na publicação disponível sobre esse estudo não há menção ao determinante movimento $(\mathrm{M})$.

A partir dessas informações, por vezes contraditórias, é que se propôs investigar em que medida o teste de Zulliger, com características semelhantes ao Rorschach, pode contribuir para a identificação da criatividade em crianças, visando ampliar os estudos que tragam evidências de validade para essa técnica nos processos de avaliação psicológica de crianças.

Este estudo teve como objetivo verificar se os indicadores cognitivos e afetivos no Teste de Zulliger que, por hipótese, se correlacionam com a criatividade, contribuem para diferenciar crianças com diferentes potenciais criativos, identificadas pelo Teste de Criatividade Figural Infantil.

\section{Método}

\section{Participantes}

Participaram do estudo 90 crianças de ambos os sexos, que cursavam a quinta série do Ensino Fundamental de escolas públicas localizadas em uma cidade do interior de São Paulo. A amostra foi selecionada por conveniência, sendo dividida em dois grupos extremos.

Grupo 1: O grupo, com baixo nível de criatividade, foi formado por 26 indivíduos, dos quais $73,1 \%$ com 11 anos, e 26,9\% com 12 anos. Nesse grupo, $46,2 \%$ dos participantes eram do sexo feminino. Como critério de inclusão nesse grupo, foram selecionados os indivíduos que obtiveram, no Teste de Criatividade Figural Infantil (TCFI) (Nakano, Wechsler, \& Primi, 2011), percentil classificado, por meio das tabelas de série e escola, como "abaixo da média" e (entre 20 e 34) e "inferior" (abaixo de 20), indicando a necessidade do desenvolvimento de características criativas.

Grupo 2: O grupo, com alto nível de criatividade, foi formado por 35 indivíduos, dos quais $49294,3 \%$ com 11 anos, e 5,7\% com 12 anos. Nesse grupo, 68,6\% dos participantes eram do gênero feminino. Conforme o Manual do TCFl, esse grupo foi classificado como "superior" (percentil acima de 81) e "acima da média" (entre 65 e 80), sugerindo indivíduos que possuíam características bem desenvolvidas quanto à criatividade.

\section{Instrumentos}

Teste de Criatividade Figural Infantil

O Teste de Criatividade Figural Infantil permite a avaliação da criatividade por meio de desenhos. Na atividade 1, a criança é convidada a completar um desenho a partir de um estímulo não definido, enquanto a atividade 2 é formada por figuras que devem ser aperfeiçoadas, e a atividade 3 propõe que a criança faça o maior número de desenhos utilizando um mesmo estímulo. É necessário que o aplicador realize a contagem do tempo, diferente para cada atividade: 5 minutos na primeira, 10 na segunda, e 10 na terceira. O tempo estimado na aplicação do TCFI é de aproximadamente 40 minutos (Nakano, et al. 2011).

Teste de Zulliger (SC)

O Teste de Zulliger é um instrumento pertencente ao grupo das técnicas projetivas, com o objetivo de gerar informações sobre a personalidade, indicando não apenas as dificuldades, mas também as potencialidades que o indivíduo possui (Franco, 2009). O teste é formado por três cartões de $24 \times 17$ centímetros, cada qual com uma mancha de tinta. A aplicação do Zulliger é feita em duas etapas: na primeira, chamada fase de resposta, o indivíduo deve responder à pergunta: "Com que isso se parece?" após visualizar cada prancha. Em seguida, tem lugar a fase do inquérito, na qual o aplicador investiga como, a partir da mancha, a criança elaborou determinada ideia. Essas informações permitem a codificação e interpretação adequada das respostas, considerando tanto os aspectos quantitativos como os qualitativos. 


\section{Procedimentos}

Foi estabelecido contato com a direção das escolas e apresentada uma carta de autorização para realização da pesquisa na instituição. Em seguida, o estudo foi submetido ao Comitê de Ética em Pesquisa da Universidade São Francisco. Após aprovação, foi apresentado o objetivo da pesquisa aos alunos e entregue a eles o Termo de Consentimento Livre e Esclarecido (TCLE). Assinaram o TCLE os pais que consentiram na participação de seus filhos. Com o recebimento dos termos assinados, foram agendadas as datas de aplicação com os diretores das escolas.

No Termo de Consentimento Livre e Esclarecido também foi informado que a aplicação dos instrumentos possuía duas etapas. Primeiramente foi aplicado de forma coletiva o TCFI, com duração de aproximadamente 30 minutos. Posteriormente a essa etapa de aplicação, foram analisados os resultados obtidos e selecionadas as crianças com os resultados extremos, ou seja, com alta criatividade (pontuação superior a 65 no TCFI) e com baixa criatividade (pontuação inferior a 34). Numa segunda etapa, foi aplicado o Teste de Zulliger (SC), com duração de aproximadamente 40 minutos. Para a comparação dos dois grupos, foram considerados inicialmente os determinantes mais mencionados na literatura - $M, R$ e P. Além deles, consideraram-se outros que se referem, no Sistema Compreensivo, à capacidade de síntese e atividade organizativa: os indicadores qualidade evolutiva (DQ+) e frequência de pontuação Z (Zf), que vão além do que a frequência simples das respostas global (W) poderia indicar. No que diz respeito à afetividade, mencionada na literatura como fator relevante na criatividade, optou-se por verificar possíveis diferenças no índice coeficiente afetivo (Afr) que registra a proporção de respostas dadas à prancha colorida do Zulliger.

\section{Resultados}

Com o intuito de averiguar a precisão da codificação do Teste de Zulliger, $25 \%$ dos protocolos foram sorteados e recodificados por um juiz inde- pendente, para realizar o estudo de precisão por meio do Coeficiente Kappa. Neste estudo, pode-se constatar que o valor mínimo de concordância foi de 0,88 e o máximo foi de 1,00; sendo assim, pode-se verificar que as variáveis selecionadas para este estudo obtiveram valores considerados bons e excelentes.

A seguir, procedeu-se às análises comparativas entre os grupos, considerando-se os indicadores selecionados para esta pesquisa.

A Tabela 1 apresenta as estatísticas do teste $t$ de Student, $d$ de Cohen relativos às variáveis utilizadas neste estudo e a comparação das respectivas médias do grupo de crianças com baixo nível de criatividade (grupo 1) e com alto nível de criatividade (grupo 2). Os resultados apontaram diferenças significativas nas variáveis $R, M$ e $P$. A variável $R$ mostrou diferença significativa entre os grupos $(p \leq 0,00)$, apresentando resultados maiores no grupo 2. A variável $\mathrm{M}$ apresentou uma diferença significativa $(p \leq 0,00)$ comparando-se os dois grupos, revelando que o grupo com alto nível de criatividade apresenta valores mais altos que o grupo com baixa criatividade. Já as respostas populares foram consideradas mais frequentes no grupo 2 e estatisticamente significativas.

No entanto, não basta que os resultados tenham diferenças estatisticamente significativas, sendo necessário também verificar a magnitude do efeito dessa diferença. As variáveis que foram significativas e as que tiveram uma magnitude considerada grande foram $\mathrm{M}(d=0,88)$ e $\mathrm{P}(d=0,89)$.

Por outro lado, em uma análise exploratória observou-se que os indicadores de qualidade formal apresentaram diferenças significativas, conforme pode ser observado a seguir.

Na Tabela 2, pode-se verificar que a variável qualidade formal incomum (FQ-) apresentou diferença significativa nos grupos $(p \leq 0,00)$, sendo maior no grupo 2. Quanto à quantidade de movimentos humanos ativos (Ma) e passivos (Mp), observa-se uma diferença significativa entre os grupos, sendo esse percentil mais elevado entre as crianças do grupo de maior criatividade $(p \leq 0,00)$. Assim, pode-se constatar que Ma $(d=0,80)$ e FQ- $(d=0,97)$ apresentaram magnitude considerada grande. 
Tabela 1

Análise comparativa das médias das variáveis selecionadas no Teste de Zulliger

\begin{tabular}{|c|c|c|c|c|c|c|c|}
\hline Variáveis & Grupos & $N$ & Média & DP & $t$ & $d$ & $p$ \\
\hline \multirow[t]{2}{*}{$\mathrm{R}$} & 1 & 26 & 4,31 & 0,73 & $-2,70$ & 0,63 & $0,001^{*}$ \\
\hline & 2 & 35 & 5,34 & 2,07 & & & \\
\hline \multirow[t]{2}{*}{ W } & 1 & 26 & 1,27 & 0,77 & $-0,62$ & 0,20 & 0,530 \\
\hline & 2 & 35 & 1,43 & 1,11 & & & \\
\hline \multirow[t]{2}{*}{$\mathrm{DQ}+$} & 1 & 26 & 0,85 & 0,88 & $-1,30$ & 0,33 & 0,190 \\
\hline & 2 & 35 & 1,14 & 0,87 & & & \\
\hline \multirow[t]{2}{*}{ Zf } & 1 & 26 & 1,85 & 1,04 & $-0,61$ & 0,16 & 0,540 \\
\hline & 2 & 35 & 2,03 & 1,22 & & & \\
\hline \multirow[t]{2}{*}{$\mathrm{M}$} & 1 & 26 & 0,30 & 0,47 & $-3,33$ & 0,88 & $0,001^{*}$ \\
\hline & 2 & 35 & 0,80 & 0,63 & & & \\
\hline \multirow[t]{2}{*}{$P$} & 1 & 26 & 0,54 & 0,70 & $-3,40$ & 0,89 & $0,001^{*}$ \\
\hline & 2 & 35 & 1,37 & 1,08 & & & \\
\hline \multirow[t]{2}{*}{ Afr } & 1 & 26 & 0,49 & 0,29 & $-0,60$ & 0,15 & 0,550 \\
\hline & 2 & 35 & 0,53 & 0,23 & & & \\
\hline
\end{tabular}

Nota: " $p \leq 0,01$.

R: Total de Respostas; W: Resposta Global; DQ+: Resposta Sintetizada; Zf: Frequência de Pontuação Z; M: Movimento Humano; P: Resposta Popular; Afr: Quociente Afetivo; DP: Desvio-Padrão.

Tabela 2

Análise comparativa das médias das variáveis no Teste de Zulliger

\begin{tabular}{|c|c|c|c|c|c|c|c|}
\hline Variáveis & Grupos & $N$ & Média & DP & $t$ & $d$ & $p$ \\
\hline \multirow[t]{2}{*}{ FQ- } & 1 & 26 & 0,50 & 0,64 & $-3,74$ & 0,97 & $0,001^{*}$ \\
\hline & 2 & 35 & 1,40 & 1,09 & & & \\
\hline \multirow[t]{2}{*}{$\mathrm{FQu}$} & 1 & 26 & 1,62 & 1,20 & 1,68 & 0,45 & 0,090 \\
\hline & 2 & 35 & 1,11 & 1,11 & & & \\
\hline \multirow[t]{2}{*}{ FQo } & 1 & 26 & 2,12 & 1,36 & $-1,57$ & 0,40 & 0,120 \\
\hline & 2 & 35 & 2,74 & 1,65 & & & \\
\hline \multirow[t]{2}{*}{$\mathrm{Mp}$} & 1 & 26 & 0,04 & 0,19 & $-0,72$ & 0,20 & 0,460 \\
\hline & 2 & 35 & 0,09 & 0,28 & & & \\
\hline \multirow[t]{2}{*}{$\mathrm{Ma}$} & 1 & 26 & 0,27 & 0,45 & $-2,93$ & 0,80 & $0,001^{*}$ \\
\hline & 2 & 35 & 0,71 & 0,66 & & & \\
\hline
\end{tabular}

Nota: ${ }^{*} p \leq 0,01$.

FQ: Qualidade Formal Menos; FQu: Qualidade Formal Incomum; FQo: Qualidade Formal Ordinária; Mp: Movimento Humano Passivo; Ma: Movimento Humano Ativo; DP: Desvio-Padrão.

\section{Discussão}

A avaliação psicológica na infância, conforme já mencionado, deve ser compreendida como um processo que permite uma adequada compreensão da criança. Uma avaliação precoce, associada a uma intervenção apropriada, aumenta a probabilidade de a criança se desenvolver de forma saudável (Nascimento et al., 2009; Pérez-Ramos, 2000). Sendo assim, é essencial o desenvolvimento de pesquisas na área da avaliação da personalidade

494 infantil, pois, com base nos resultados, essas crian- ças poderão ser encaminhadas para tratamento apropriado. Entretanto, são escassos no Brasil os instrumentos aprovados para avaliação de crianças, o que justifica o investimento em pesquisas nessa área. O teste de Zulliger tem se mostrado útil na avaliação de pessoas adultas e, dada sua semelhança com o Rorschach, é possível esperar que seja também útil na avaliação de crianças, mas isso ainda não foi suficientemente demonstrado.

A produção de respostas no Teste de Zulliger abarca uma grande quantidade de processos psico- 
lógicos, tais como atenção, percepção, tomada de decisão, análise lógica, e, por isso partiu-se do pressuposto de que ele poderia ser útil na avaliação da criatividade, tal como postulado por alguns autores em relação ao Método de Rorschach (Sakamoto, Lapastini, \& Silva, 2003; Weiner, 2000).

Considerando-se dois grupos de crianças com desempenhos extremos num teste de criatividade, pode-se observar que a variável número de respostas (R) está relacionada com tendências mais ou menos criativas, além de outras características da personalidade que colaboram para essa produção.

De acordo com o esperado, contatou-se que a variável $R$ diferiu significativamente entre os grupos e que as crianças com alto nível de criatividade apresentaram maior quantidade de respostas ao Zulliger. Esse dado confirma o achado de Singh et al., (2007), que encontraram no Rorschach um número maior de respostas em pessoas mais criativas.

No que diz respeito à capacidade de síntese e atividade de pensamento organizativo, não se encontrou neste estudo diferença significativa em relação à frequência de $W$. Ampliando a possibilidade de avaliar esses pressupostos no teste de Zulliger, o Sistema Compreensivo inclui em sua análise os índices DQ+ e Zf, estudados nesta pesquisa. Porém, nenhuma dessas duas variáveis mostrou diferença significativa. Embora os números absolutos apontem uma frequência maior de ambas nas crianças mais criativas, isso só poderia ser confirmado com amostras maiores. Sendo assim, essas variáveis não revelaram o desempenho esperado e não confirmaram, neste estudo, os achados de Singh et al., (2007).

As respostas de movimento humano têm sido aquelas que mais frequentemente são indicadas na literatura como relacionadas com a criatividade. O grupo das crianças mais criativas teve maior média de $\mathrm{M}$ em relação às crianças com baixa criatividade. Pode-se verificar que a diferença entre os grupos foi considerada estatisticamente significativa, e que o determinante M mostrou-se sensível para diferenciar níveis de criatividade.

Apesar de o estudo de Singh et al., (2007) apontar o aumento de $\mathrm{P}$ em pessoas mais criativas, esse dado parecia um pouco contraditório devido ao fato de P estar associado ao senso comum. Sendo assim, como hipótese inicial deste estudo com o Teste de Zulliger, esperava-se que as crianças menos criativas apresentassem mais respostas populares (P). No entanto, o resultado mostrou o contrário, com aumento da média de $\mathrm{P}$ no grupo das crianças mais criativas, o que vai de encontro aos autores citados. Mas, além disso, é importante ressaltar que, na prancha III, aparecem três das cinco respostas populares do Zulliger, sendo duas de conteúdo humano - valendo salientar que essa prancha é responsável pelo aumento das respostas de movimento humano. Tal fato acarreta uma frequência maior de respostas populares, principalmente quando maior for a propensão de a pessoa ver figuras humanas em movimento.

Embora, com base nos dados de literatura, não tenha sido prevista a adequação perceptiva representada pelas variáveis de qualidade formal ordinária (FQo), qualidade formal incomum (FQu) e qualidade formal distorcida (FQ-) -, revelou-se um resultado importante neste estudo. A frequência de FQ- foi significativamente maior no grupo das crianças com alto nível de criatividade. Esse resultado sugere que o grupo das crianças mais criativas estava mais propenso a uma visão excessivamente subjetiva, por vezes distorcida da realidade. Esse dado pode isoladamente levar a pensar que se trata de crianças mais desadaptadas do que se esperaria, porém tal conclusão só seria possível se fosse possível demonstrar que as crianças que apresentaram mais FQ- também tivessem menor frequência de $\mathrm{FQo} \mathrm{ou} \mathrm{FQu} \mathrm{em} \mathrm{relação} \mathrm{às} \mathrm{normas} \mathrm{esperadas.} \mathrm{Esse}$ dado não foi possível verificar, uma vez que ainda não estão disponíveis as tabelas normativas do Zulliger para crianças.

Segundo Wechsler (1993), a flexibilidade de ideias, compreendida como a capacidade de mudar de ponto de vista de acordo com a necessidade ou diante de um problema, está profundamente associada à manifestação da criatividade. Nessa mesma direção, Weiner (2000) afirma que o pensamento flexível contribui para o bom ajustamento. Os resultados deste trabalho evidenciaram que o grupo de crianças com alto nível de criatividade 
apresentou respostas que indicam flexibilidade do pensamento, constatado pelo equilíbrio satisfatório de movimentos ativos e passivos ( $a=p$ maior em crianças criativas com $p=0,001$ ). Essas duas afirmações podem ajudar a compreender que o simples aumento de FQ- nas crianças mais criativas poderia demonstrar uma flexibilidade maior, desde que as frequências não escapassem aos padrões normativos.

Por fim, em relação aos aspectos emocionais envolvidos na criatividade, Sakamoto (2000) afirma que, desde o início da vida, as relações afetivas e o ambiente possibilitam que o indivíduo desenvolva o sentimento de segurança, condição essencial para a manifestação criativa. Sendo assim, a forma como o sujeito vivencia suas emoções influencia em diversos aspectos, abrangendo também os que se relacionam com a capacidade de criar (Villemor-Amaral \& Primi, 2009; Barreto e Martinez, 2007).

Nessa mesma direção, Sakamoto et al. (2003) asseguram que o envolvimento afetivo está associado à realização criativa. Desse modo, a variável coeficiente afetivo (Afr) foi analisada neste estudo, visando verificar se a responsividade ao estímulo afetivo se modifica conforme os níveis de criatividade medida pelo TCFI. Um protocolo com Afr aumentado indica que o indivíduo se deixa atrair pela estimulação emocional, demonstrando ser bastante produtivo nessas ocasiões. Embora a média do grupo das crianças com alto nível de criatividade tenha sido maior, esse aumento não foi estatisticamente significativo. Sendo assim, pode-se verificar apenas uma tendência a responder com mais envolvimento nas situações carregadas emocionalmente, não constituindo, até o momento, uma variável segura para aferir a criatividade.

Considerando-se que o objetivo deste estudo foi verificar se os indicadores cognitivos e afetivos no Teste de Zulliger contribuem para identificar crianças com diferentes potenciais criativos identificadas pelo Teste de Criatividade Figural Infantil, pode-se constatar que os dados apresentados confirmaram que o primeiro teste é um instrumento que permite diferenciar grupos com resultados extremos no segundo. Isso foi constatado por meio 496 de diferenças significativas em indicadores que, na literatura sobre o Método de Rorschach, já haviam sido considerados relevantes para compreensão da criatividade.

Tal fato indica que o Zulliger pode ser útil para avaliação de crianças, embora seja desejável que se acumulem outras evidências relacionadas com diversos aspectos da personalidade infantil. Por fim, espera-se a realização de novos estudos sobre as qualidades psicométricas do Teste de Zulliger, dada a sua reconhecida importância dentre os instrumentos de avaliação da personalidade, de modo a permitir seu uso mais seguro nos diversos contextos em que seja necessário maior conhecimento sobre a criança, fornecendo subsídios para orientações e encaminhamentos mais acertados.

\section{Referências}

Adrados, I. (1978). Teoria e prática do teste de Rorschach. Petrópolis: Vozes.

Alencar, E. M. S., \& Fleith, D. S. (2003). Barreiras à criatividade pessoal entre professores de distintos níveis de ensino. Psicologia: Reflexão e Crítica, 16(1), 63-69.

Aronow, E., \& Raychaudhuri, M. (1972). Comment on Raychaudhuri's relation on creativity and sex to Rorschach $\mathrm{M}$ responses. Journal of Personality Assessment, 36(4), 303-306.

Barreto, M. O., \& Martinez, A. M. (2007). Possibilidades criativas de professores em cursos de pós-graduação stricto sensu. Estudos de Psicologia (Campinas), 24(4), 463-473

Dudek, S. Z. (1968). M an active energy system correlating Rorschach $M$ with ease of creative expression. Journal of Projective Techniques and Personality Assessment, 32(5), 453-461.

Franco, R. R. C. (2009). Ensaio de convergência entre provas de personalidade: Zulliger-SC e Pfister (Tese de doutorado não-publicada). Universidade São Francisco, Itatiba.

Exner, J. E., \& Sendín, C. (1999). Manual de interpretação do Rorschach para o sistema compreensivo. São Paulo: Casa do Psicólogo.

Joseph, A., \& Pillai, A. (1986). Projective indices of creativity. Indian Journal of Clinical Psycholog, 13(1), 9-13.

Nakano, T. C., Wechsler, S. M., \& Primi, R. (2011). Teste de Criatividade Figural Infantil. São Paulo: Vetor.

Nascimento, R., Pedroso, J. S., \& Souza, A. M. (2009). Método de Rorschach na avaliação psicológica em crianças: uma revisão de literatura. Psico-USF, 14(2), 193-200. 
Oliveira, E. B. P., \& Alencar, E. M. L. S. (2012). Importância da criatividade na escola e no trabalho docente segundo coordenadores pedagógicos. Estudos de Psicologia (Campinas), 29(4), 541-552.

Pérez-Ramos, A. L. M. Q. (2000). Avaliação prospectiva: o exame precoce da criança. In Cunha, J. A. (Org.), Psicodiagnóstico - V (pp.151-157). Porto Alegre: Artmed.

Raychaudhuri, M. (1971). Relation of creativity and sex to Rorschach $M$ responses. Journal of Personality Assessment, 35(1), 27-31.

Rorschach, H. (1978). Psicodiagnóstico de Rorschach. São Paulo: Mestre Jou.

Sakomoto, C. K. (2000). Criatividade: uma visão integradora. Psicologia: Teoria e Prática, 2(1), 50-58.

Sakamoto, C. K., Lapastini, M. B., \& Silva, S. M. (2003). A criatividade no psicodiagnóstico de Rorschach: uma possibilidade de enriquecimento à interpretação dos resultados. Psicologia: Teoria e Prática, 5(1), 13-25.

Singh, D. K., Majhi, G., \& Singh, A. R. (2007). Projective indices of creativity: A pilot study. Journal of Projective Psychology \& Mental Health, 14(2) 117-120.

Torres, J. M. A. (2010). A resposta de movimento humano nos protolocos de Rorschach de artistas pintores (Dissertação de mestrado não-publicada). Universidade Federal do Pará, Belém.
Villemor-Amaral, A. E. (2006). Desafios para a cientificidade das técnicas projetivas. In A. P. P. Noronha, A. A. A. Santos, \& F. F. Sisto (Orgs.), Facetas do fazer em Avaliação Psicológica (pp.163-171). São Paulo: Vetor.

Villemor-Amaral, A. E. (2008). A Validade Teórica em avaliação psicológica. Psicologia Ciência e Profissão, 28(1), 98-109.

Villemor-Amaral, A. E., \& Pasqualini-Casado, L. (2006). A cientificidade das técnicas projetivas em debate. Psico-USF, 11(2), 185-193.

Villemor- Amaral, A. E., \& Primi, R. (2009). Teste de Zulliger no Sistema Compreensivo ZSC - forma individual. São Paulo: Casa do Psicólogo.

Wechsler, S. M. (1993). Criatividade: descobrindo e encorajando. São Paulo: Editora Psy.

Wechsler, S. M. (1998). Criatividade: descobrindo e encorajando. São Paulo: Editora Psy.

Wechsler, S. M. (2001). Criatividade na cultura brasileira: uma década de estudos. Revista Portuguesa de Psicologia: Teoria, Investigação e Prática, 6(1) 215-227.

Weiner, I. (2000). Princípios da interpretação do Rorschach. São Paulo: Casa do Psicólogo.

Recebido em: 1/2/2013

Versão final em: $27 / 5 / 2013$

Aprovado em: 12/9/2013 
